\title{
Hook Plate Fixation for the Thumb Ulnar Collateral Ligament Fracture-Avulsion
}

\author{
Ali Tabrizi ${ }^{1} \quad$ Ahmadreza Afshar $^{1}$ \\ ${ }^{1}$ Department of Orthopedics, Urmia University of Medical Sciences, \\ Urmia, Iran \\ J Hand Microsurg 2017;9:95-97.

\begin{abstract}
Address for correspondence Ahmadreza Afshar, MD, Department of Orthopedics, Urmia University of Medical Sciences, Imam Khomeini Hospital, Modaress Street, Ershad Boulevard, Urmia, 5715781351 , Iran (e-mail: afshar_ah@yahoo.com).
\end{abstract}
Abstract
Keywords
- fracture-avulsion
- hook plate fixation
- skiers' thumb
- thumb ulnar collateral ligament

During a taekwondo competition, a 20-year-old female competitor injured her left thumb. There was pain, swelling, and tenderness at the ulnar side of the metacarpophalangeal joint. Plain radiography demonstrated an avulsion fracture with a small-size fragment at the base of the proximal phalanx. A mini hook plate was used to repair the lesion. The patient was satisfied with the results and returned to her sports activities after 2 months.

\section{Introduction}

Acute ulnar collateral ligament (UCL) injury to the thumb metacarpophalangeal (MCP) joint is a common injury, particularly among athletes. This injury is produced by a forceful abduction and radial deviation of the thumb MCP joint. The injured UCL may result in instability of the thumb MCP joint, pinch and grasp weaknesses, and, in the long term, may predispose the joint to progressive secondary osteoarthritis. ${ }^{1-10}$

Usually, disruption of the UCL occurs at the distal attachment of the ligament. However, in 20 to $50 \%$ of the patients, the thumb UCL avulsion may be accompanied with a fracture-avulsion from the base of the thumb proximal phalan $x^{2,3}$ Displacement of more than $2 \mathrm{~mm}$ or rotation of the fragment needs repair of the ligament and open reduction of the fragment. ${ }^{3}$

Several techniques have been used for repair of UCL fracture-avulsions. ${ }^{1-10} \mathrm{~A}$ hook plate has been used successfully for treatment of various small avulsion fractures in the hand. ${ }^{8,11}$ Hook plates provide a stable fixation to begin early range of motion (ROM) ${ }^{8,11}$ In this case, the authors use a mini hook plate to treat thumb UCL fracture-avulsion. To the authors' best knowledge, this case is the first in vivo documented experience of this technique.

\section{Case Report}

A 20-year-old female taekwondo player complained of pain in her left thumb due to a direct impact during competition.
There was pain, swelling, and tenderness at the ulnar side of the thumb MCP joint. Plain radiography demonstrated a small fracture-avulsion at the base of the proximal phalanx of the thumb (-Fig. 1). A valgus instability test was not performed due to the risk of displacement of the avulsed fragment and potential development of a Stener's lesion. Under general anesthesia, the lesion was approached through a standard lazy S dorsoulnar approach centered at the thumb MCP joint. The radial superficial nerve was found and protected. The adductor aponeurosis was incised parallel to the extensor pollicis longus tendon. The avulsed fragment was identified. Open reduction of the avulsed fragment was performed, and a low-profile 2-mm stainless steel hook plate with one $2-\mathrm{mm}$ screw was used for the internal fixation. The plate was fashioned from a longer $2-\mathrm{mm}$ plate, trimming a one and half-hole plate with hook extensions on the proximal side of the plate to wrap around and grasp the avulsed fragment. The fashioned plate was fixed with one $2-\mathrm{mm}$ screw inserted through the single hole of the plate. The reduction was checked by an image intensifier. The rupture in the dorsal capsule was identified and repaired. At surgery, a valgus stress test of the thumb MCP joint was performed and the result showed the joint to be completely stable (-Fig. 2). A spica thumb cast was applied for 2 weeks; then, the thumb was protected by a thermoplastic thumb splint for 6 weeks. At 2 months, plain radiography demonstrated satisfactory osseous healing (-Figs. 3, 4). The thumb MCP was completely stable; however, there was mild pain
DOI https://doi.org/ 10.1055/s-0037-1604292. ISSN $0974-3227$. (c) 2017 Society of Indian Hand \& Microsurgeons 


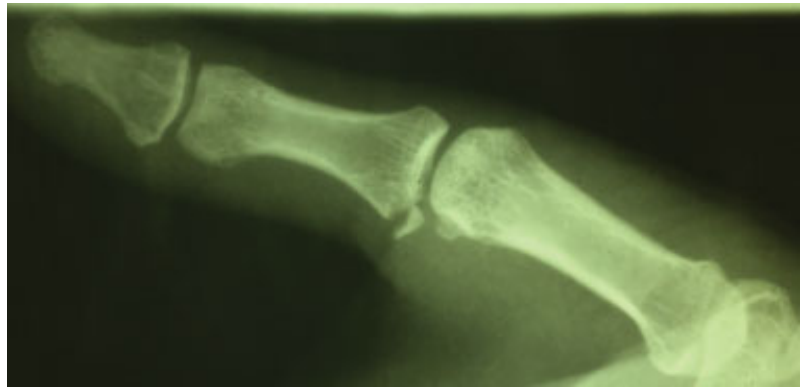

Fig. 1 Plain radiography of the left thumb demonstrates the $\mathrm{UCL}$ fracture-avulsion lesion.

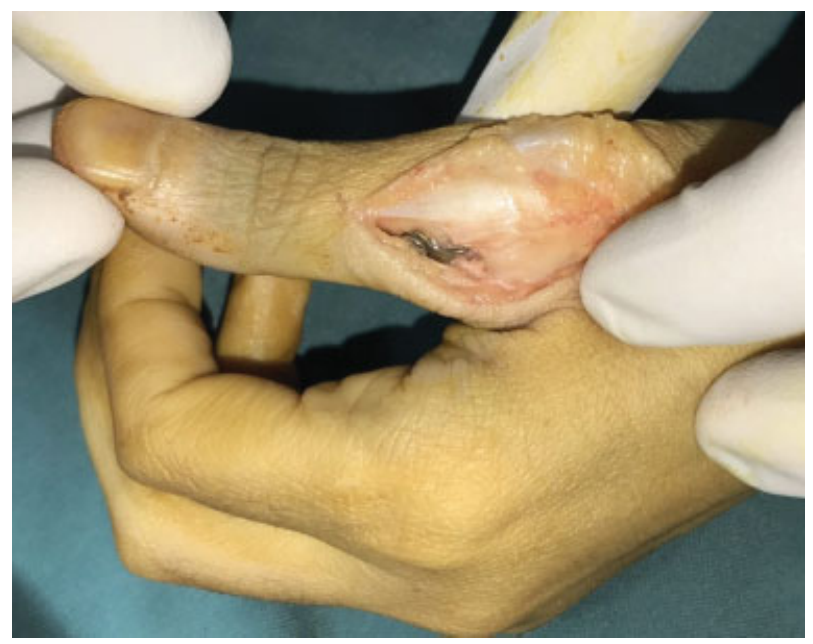

Fig. 2 Valgus stress test of the thumb MCP joint was completely stable at the end of the surgery.

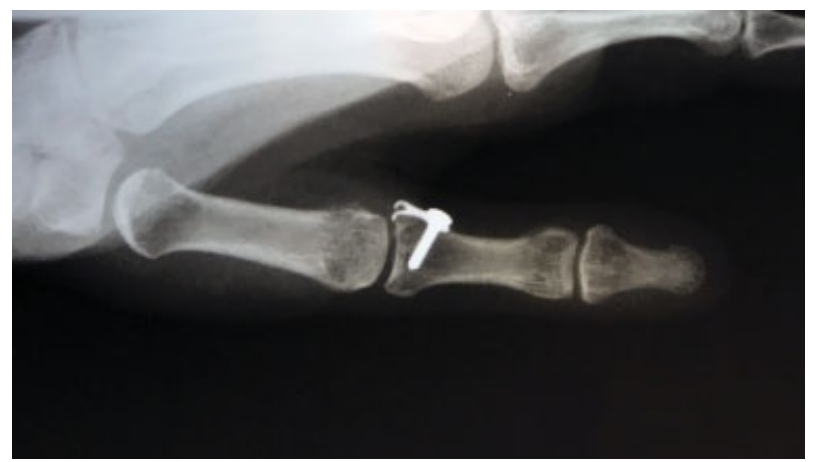

Fig. 3 Plain radiography of the left thumb 2 months post surgery demonstrates complete healing of the fracture-avulsed fragment.

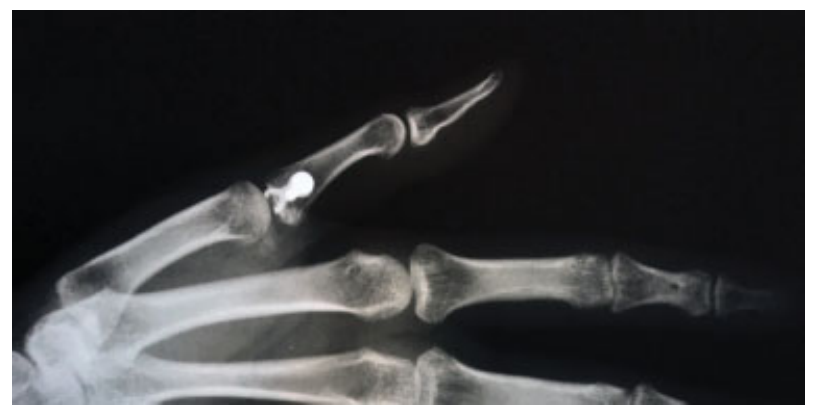

Fig. 4 Lateral radiograph of the left thumb MCP joint 2 months post surgery. and swelling at the thumb MCP joint. The key pinch and power grip strengths of the right hand were $6 \mathrm{~kg}$ and $40 \mathrm{~kg}$ whereas the left hand's were $4.5 \mathrm{~kg}$ and $25 \mathrm{~kg}$, respectively, at 2 months post surgery. The patient was satisfied with the results and returned to her sports activities with a splint protection after 2 months. The plate did not disturb her during her activities; therefore, she had no intention to remove the plate.

\section{Discussion}

Though undisplaced fracture-avulsion the thumb UCL injuries can be treated nonsurgically with casting and immobilization, several techniques have been suggested for the repair of the displaced and rotated fracture-avulsion the thumb UCL injuries, including fixation with screw or pin, cerclage wire, ${ }^{2}$ transosseous sutures tied over a bone tunnel or pull-out sutures over a button on the radial aspect of the MCP joint, ${ }^{3,10}$ suture anchor, ${ }^{5,8,9}$ tension band wiring, ${ }^{4}$ excision of the small fragment, and direct repair of the ligament. ${ }^{3,7}$

Each of these techniques has disadvantages. For example, placement of a button on the radial aspect of the MCP joint may cause skin irritation and problems. Tension band wiring does not produce a force vector perpendicular to the fracture plane; therefore, it predisposes the small fracture-avulsion fragment to rotate. ${ }^{3}$ Regarding the size of the avulsed osseous fragment, screw and pin may be used for a relatively large fragment. However, it may not be possible to directly fix a small-size fragment with a mini screw or pin. ${ }^{10}$

A suture anchor is a favorable method of the thumb UCL fracture-avulsion treatment. ${ }^{5,8,9}$ One of the advantages of this method is that there is no need to externally pull out wires or buttons. The technique uses one or two $1.3-$ or $1.5-\mathrm{mm}$ mini anchors. ${ }^{5}$ Katolik et al found shorter surgical time, lower cost, and decreased soft tissue complications by using suture anchors versus pull-out sutures. ${ }^{6}$

In a cadaveric study, Shin et al compared hook plate fixation with suture anchor fixation in thumb UCL fracture-avulsions. In their study, hook plate fixation failed at a significantly higher force (average $58 \mathrm{~N}$ ) than the suture anchor fixation (average $27 \mathrm{~N}$ ). The suture anchor is placed in the cancellous bone, which may not provide a sturdy fixation whereas the hook plate is fixed with a bicortical screw in an unfractured bone. In this study, hook plates provided a higher stability than the suture anchor for UCL fractureavulsions. Application of suture anchors for a small bone fragment does not guarantee a precise reduction because it may produce a rotational momentum, ${ }^{3,8}$ whereas application of a hook plate provides a more precise reduction of the fragment. ${ }^{8}$

To the authors' best knowledge, application of a hook plate in thumb UCL fracture-avulsion in vivo has not been reported. In this case, the cost of treatment with a fashioned stainless steel hook plate was not higher than that of the other techniques, and application of hook plate for thumb UCL fractureavulsion resulted in a satisfactory healing, complete stability of the thumb MCP joint, and return to sport activities after 2 months post surgery. 


\section{Conflict of Interest}

None.

\section{References}

1 Bovard RS, Derkash RS, Freeman JR. Grade III avulsion fracture repair on the UCL of the proximal joint of the thumb. Orthop Rev 1994;23(02):167-169

2 Carlsen BT, Moran SL. Thumb trauma: Bennett fractures, Rolando fractures, and ulnar collateral ligament injuries. J Hand Surg Am 2009;34(05):945-952

3 Dinowitz M, Trumble T, Hanel D, Vedder NB, Gilbert M. Failure of cast immobilization for thumb ulnar collateral ligament avulsion fractures. J Hand Surg Am 1997;22(06):1057-1063

4 Jupiter JB, Sheppard JE. Tension wire fixation of avulsion fractures in the hand. Clin Orthop Relat Res 1987;(214):113-120

5 Moharram AN. Repair of thumb metacarpophalangeal joint ulnar collateral ligament injuries with microanchors. Ann Plast Surg 2013;71(05):500-502
6 Katolik LI, Friedrich J, Trumble TE. Repair of acute ulnar collateral ligament injuries of the thumb metacarpophalangeal joint: a retrospective comparison of pull-out sutures and bone anchor techniques. Plast Reconstr Surg 2008;122(05): 1451-1456

7 Pulos N, Shin AY. Treatment of ulnar collateral ligament injuries of the thumb: a critical analysis review. JBJS Rev 2017;5(02):21

8 Shin EH, Drake ML, Parks BG, Means KR Jr. Hook plate versus suture anchor fixation for thumb ulnar collateral ligament fracture-avulsions: a cadaver study. J Hand Surg Am 2016;41(02): 192-195

9 Tsiouri C, Hayton MJ, Baratz M. Injury to the ulnar collateral ligament of the thumb. Hand (NY) 2009;4(01):12-18

10 Weiland AJ, Berner SH, Hotchkiss RN, McCormack RR Jr, Gerwin M. Repair of acute ulnar collateral ligament injuries of the thumb metacarpophalangeal joint with an intraosseous suture anchor. J Hand Surg Am 1997;22(04):585-591

11 Kang GC, Yam A, Phoon ES, Lee JY, Teoh LC. The hook plate technique for fixation of phalangeal avulsion fractures. J Bone Joint Surg Am 2012;94(11):e72 